\title{
O EMPREGO DA TERMOGRAVIMETRIA PARA DETERMINAR A ENERGIA DE ATIVAÇÃO DO PROCESSO DE COMBUSTÃO DE ÓLEOS COMBUSTÍVEIS
}

\author{
Cecilia Rocío Morales Leiva*, Paula Manoel Crnkovic e Antonio Moreira dos Santos \\ Departamento de Engenharia Mecânica, Escola de Engenharia de São Carlos, Universidade de São Paulo, \\ CP 359, 13560-970 São Carlos - SP, Brasil
}

Recebido em 6/6/05; aceito em 11/11/05; publicado na web em 6/7/06

\begin{abstract}
THE EMPLOYMENT OF THERMOGRAVIMETRY TO DETERMINE ACTIVATION ENERGY IN THE COMBUSTION PROCESS OF FUEL OILS. Activation energy (Ea) is a parameter that can be applied to make predictions about the quality of oils to be used in an ICO engine. In this study, Ea was determined by thermogravimetry following two different procedures: ASTM E 1641 and Model-free kinetics. The energies were calculated in the low temperature oxidation (LTO) region for three Brazilian fuel oils (denominated A, B and C) and the results were equal using both methods: $43 \mathrm{~kJ} \mathrm{~mol}^{-1}\left(\alpha=0.1\right.$ to 0.9 ) for oil A, $48 \mathrm{~kJ} \mathrm{~mol}^{-1}$ $(\alpha=0.1$ to 0.5$)$ and $65 \mathrm{~kJ} \mathrm{~mol}^{-1}(\alpha=0.5$ to 0.9$)$ for oil $\mathrm{B}$, and $58 \mathrm{~kJ} \mathrm{~mol}^{-1}(\alpha=0.1$ to 0.5$)$ and $65 \mathrm{~kJ} \mathrm{~mol}^{-1}(\alpha=0.5$ to 0.9$)$ for oil $\mathrm{C}$. It was concluded that, among the oils studied, sample A was potentially the best option concerning the behavior in the LTO region.
\end{abstract}

Keywords: activation energy; fuel oil; thermogravimetry.

\section{INTRODUÇÃO}

Óleos combustíveis são largamente utilizados na indústria moderna para aquecimento de fornos e caldeiras, ou em motores de combustão interna ${ }^{1}$. Dentre as várias características de um óleo combustível para motores de ignição por compressão (ICO), podese salientar a homogeneidade de queima que está relacionada com o atraso de ignição, ou seja, tanto melhor será o combustível quanto menor for o atraso de ignição (intervalo entre o início da injeção do combustível e o início da combustão). Um atraso longo provoca um acúmulo de combustível sem queimar na câmara e, ao entrar em auto-ignição, já fora do ponto ideal, provoca um fenômeno indesejável denominado "batida de pino",2, A qualidade de ignição de um combustível é comumente definida pelo seu número de cetano $^{2}$. Um maior número de cetano corresponde a um menor atraso de ignição, assegurando melhor arranque, melhor combustão, diminuição do ruído, deslocamento mais uniforme e menor emissão dos gases tóxicos.

Para óleos combustíveis a energia de ativação $\left(\mathrm{E}_{\mathrm{a}}\right)$ do processo de combustão é um dos parâmetros que também pode avaliar a qualidade de um combustível, pois está diretamente relacionada com o atraso de ignição $(\tau)$, que é uma função $(f)$ da pressão $(p)$ e da temperatura $(\mathrm{T})$, representado pela seguinte relação ${ }^{4}$ :

$$
\tau=f\left(\mathrm{e}^{-\mathrm{E}_{\mathrm{a}} / \mathrm{RT}} / \mathrm{p}^{\mathrm{b}}\right)
$$

sendo $\mathrm{R}$ a constante universal dos gases, $\mathrm{E}_{\mathrm{a}}$ energia de ativação e $\mathrm{b}$ o coeficiente cinético.

Em motores ICO o início da combustão se dá por auto-ignição do combustível. Primeiramente, o ar é aspirado para o interior do cilindro e, em seguida, é comprimido pelo êmbolo, de forma a elevar a temperatura e a pressão da câmara de combustão. Neste ponto injeta-se o combustível que entra em auto-ignição e, devido ao aumento de pressão, o êmbolo é empurrado para baixo.
Partindo deste princípio, tanto melhor será a ignição quanto menor for o atraso de ignição do sistema e, conseqüentemente, menor a energia de ativação da combustão do óleo combustível.

Deste modo, a $\mathrm{E}_{\mathrm{a}}$ da combustão é então um parâmetro importante para se avaliar a qualidade de um combustível. Este parâmetro cinético pode ser obtido a partir de ensaios experimentais e a análise térmica é uma ferramenta útil, que possibilita essa determinação. Contudo, necessita-se definir modelos descritivos destes processos.

Há uma vasta literatura que se dedica ao emprego de técnicas de análise térmica para maior compreensão do comportamento de degradação desses derivados de petróleo ${ }^{5-15}$.

Nesses estudos, as técnicas de análise térmica mais empregadas são análise termogravimétrica (TG), análise térmica diferencial (DTA) e calorimetria exploratória diferencial (DSC) ${ }^{16}$. TG é uma técnica na qual a massa de uma substância é medida em função da temperatura, enquanto a substância é submetida a uma programação controlada de temperatura. DTA é uma técnica que consiste em medir a diferença de temperatura $(\Delta \mathrm{T})$ entre a substância e o material de referência, enquanto ambos estão sujeitos a um programa controlado de temperatura. DSC é uma técnica em que se relaciona a diferença de energia fornecida à substância e a um material de referência, em função da temperatura, enquanto a substância e o material de referência são submetidos a uma programação controlada de temperatura $^{17}$.

Métodos cinéticos tradicionais para se analisar reações de primeira ou segunda ordem não são freqüentemente aplicáveis em reações complexas e de interesse prático envolvendo, por ex., degradação térmica de óleos combustíveis. Conseqüentemente, uma variedade de técnicas matemáticas, freqüentemente denominadas de análise cinética global, foi desenvolvida para caracterizar o comportamento cinético deste tipo de reação ${ }^{18}$.

Dentre os modelos cinéticos pode-se citar os de Arrhenius, Coats e Redfern, Michelson e Eirnhorn (Ratio model), Ingraham e Marrier, Freeman e Carroll, Flynn e Wall, Ozawa e "Model-free kinetics".

Entre os precursores dos trabalhos empregando análise térmica, Tadema $^{19}$ se destaca como sendo o primeiro pesquisador que aplicou a análise térmica diferencial (DTA) no estudo dos efeitos térmicos resultantes da combustão de mistura de vários óleos crus na presen- 
ça de areia. Os experimentos mostraram duas regiões de reação e foram denominadas de oxidação à baixa e alta temperatura (LTO e HTO) e ocorrem por volta de 200 e $350{ }^{\circ} \mathrm{C}$, respectivamente.

Mais recentemente, a aplicação de técnicas de análise térmica para estudar a cinética da pirólise e da combustão de combustíveis fósseis tem alcançado ampla aceitação entre pesquisadores. Parte dessa tendência deve-se à rapidez, pequena escala e baixo custo que caracterizam os ensaios em análise térmica quando comparados aos experimentos em protótipos de sistemas de combustão em geral $^{20}$. Muitos dos trabalhos em análise térmica estão direcionados para correlacionar o comportamento térmico da amostra com a cinética ${ }^{21}$.

Ali et al. ${ }^{11}$ salientam que a termogravimetria tem sido extensivamente utilizada para caracterizar óleos crus e outros produtos derivados de petróleo.

Verkoczy e $\mathrm{Jha}^{6}$ realizaram experimentos em TG/DTG e calorimetria exploratória diferencial pressurizada (PDSC) para determinar parâmetros cinéticos de quatro óleos pesados. Dados cinéticos e termoquímicos foram estimados nas regiões de oxidação à baixa e alta temperatura, craqueamento térmico e coqueificação.

$\mathrm{Kök}^{7}$ caracterizou pirólise e comportamento termo-oxidativo de dois óleos crus utilizando TG e DSC, em atmosferas de nitrogênio e ar. Quando o óleo foi aquecido em atmosfera de nitrogênio, observaram-se duas regiões de perda de massa, a primeira referente à destilação e a segunda envolvendo degradação e craqueamento térmico. Nos ensaios envolvendo atmosfera oxidante (ar), três diferentes regiões foram identificadas para ambos os óleos, oxidação à baixa temperatura (LTO - "low temperature oxidation"), depósito de combustível (FD - "fuel deposit") e oxidação à alta temperatura (HTO "high temperature oxidation"). Para a região de HTO, os estudos cinéticos indicaram valores de energia de ativação de 128,3 e 142,3 $\mathrm{kJ} \mathrm{mol}^{-1}$ para os dois óleos avaliados. Observaram também que à medida que a energia de ativação aumentava o ${ }^{\circ} \mathrm{API}$ do óleo diminuia. Segundo o "American Petroleum Institute" o ${ }^{\circ}$ API é uma forma de expressar a densidade relativa de um óleo ou derivada. A escala varia inversamente com a densidade relativa.

Kök e Okandan ${ }^{9}$ também correlacionaram os valores de energia de ativação com o ${ }^{\circ}$ API de seis óleos crus. Os autores aplicaram método não-isotérmico com o uso de TG/DTG em excesso de ar e concluíram que estes parâmetros são inversamente proporcionais, isto é, enquanto a energia de ativação variou de 67,4 a 131,9 kJ $\mathrm{mol}^{-1}$ o ${ }^{\circ} \mathrm{API}$ variou de 26,1 a 11,3 , respectivamente.

Kök et al. ${ }^{8}$ utilizaram um analisador termogravimétrico de alta pressão (HPTG) para estudar a oxidação de três óleos crus sob condições pressurizadas (100, 200 e 300 psig). Empregaram um meio oxidante, razão de aquecimento constante $\left(10^{\circ} \mathrm{C} \mathrm{min}^{-1}\right)$ e observaram três diferentes regiões nas curvas TG. Empregaram o método de Coats e Redfern para obtenção dos parâmetros cinéticos nas regiões LTO e HTO. Os valores obtidos de energia de ativação para as três amostras na pressão de 100 psig foram de 22,6; 23,9 e
41,1 $\mathrm{kJ} \mathrm{mol}^{-1}$ para a região LTO e 99,6; 77,9 e 97,1 $\mathrm{kJ} \mathrm{mol}^{-1}$ para a região HTO. Para as demais pressões avaliadas, os autores verificaram que os parâmetros cinéticos não eram influenciados pelo efeito da pressão.

Ali et al. ${ }^{11}$ utilizaram TG e DTA para caracterizar quatro óleos crus. Essas técnicas permitiram a investigação das propriedades dos componentes voláteis desses óleos e a correlação das características termo-oxidativas. Para todas as amostras foram observadas quatro regiões características, a primeira caracterizada pela evaporação de voláteis $\left(20\right.$ a $\left.280{ }^{\circ} \mathrm{C}\right)$ e as restantes caracterizaram-se pela degradação oxidativa de diferentes classes de hidrocarbonetos, os de baixa $\left(280\right.$ a $\left.400{ }^{\circ} \mathrm{C}\right)$, média $\left(400\right.$ a $\left.510^{\circ} \mathrm{C}\right)$ e os de alta massa molar $(510$ a $620^{\circ} \mathrm{C}$ ), respectivamente. Cada óleo cru foi caracterizado por apresentar diferentes perdas de massa para cada região. Como todos os óleos crus sofrem perda de massa por aquecimento, é possível estabelecer uma diferença entre dois óleos crus com base na característica da forma de suas curvas TG.

Esse trabalho tem como objetivos a determinação da energia de ativação de uma das etapas de combustão de óleos combustíveis brasileiros por meio de análise termogravimétrica utilizando-se os métodos cinéticos ASTM E 1641 e "Model-free kinetics", a comparação dos resultados entre os métodos aplicados e o estabelecimento da energia de ativação como um parâmetro que caracteriza e qualifica os diferentes óleos.

\section{PARTE EXPERIMENTAL}

O equipamento usado para os experimentos de termogravimetria foi um sistema Shimadzu 51H. Empregaram-se as seguintes razões de aquecimento: 2,$5 ; 5,0 ; 10,0 ; 15,0$ e $20,0{ }^{\circ} \mathrm{C}$ min $^{-1}$ entre a temperatura ambiente e $600{ }^{\circ} \mathrm{C}$. Os experimentos foram feitos em duplicata para garantir boa reprodutibilidade. As demais condições experimentais foram massa da amostra de aproximadamente 20 mg, suporte de amostra de alumínio e gás de arraste ar sintético com vazão de $100 \mathrm{~mL} \mathrm{~min}^{-1}$.

Para as análises de DTA, utilizou-se o sistema Shimadzu 50 e empregou-se uma razão de aquecimento $5,0{ }^{\circ} \mathrm{C} \mathrm{min}^{-1}$, massa da amostra de aproximadamente $20 \mathrm{mg}$, suporte de amostra de alumínio e gás de arraste ar sintético com vazão de $100 \mathrm{~mL} \mathrm{~min}{ }^{-1}$.

Foram estudadas três amostras distintas cedidas pela Petrobras - Cenpes: MF-380, óleo combustível A2 e óleo combustível A1. Neste trabalho denominaram-se as amostras por A, B e C, respectivamente. A Tabela 1 apresenta as especificações e propriedades destas três amostras.

\section{Métodos cinéticos}

O método desenvolvido pelo comitê $\mathrm{ASTM}^{22}$ para determinação de parâmetros cinéticos (energia de ativação e fator pré-exponencial) por meio de dados termogravimétricos é baseado na suposição que a decomposição obedece a cinética de primeira ordem. O método

Tabela 1. Especificações e propriedades das amostras A, B e C

\begin{tabular}{lccc}
\hline & Amostra A & Amostra B & Amostra C \\
\hline Classificação(Petrobras) & MF $380(\mathrm{~A})$ & Óleo Combustível A2 & Óleo Combustível A1 \\
Viscosidade Cinemática & $\left(50{ }^{\circ} \mathrm{C}\right) 300$ e $380 \mathrm{cSt}$ & $\left(60{ }^{\circ} \mathrm{C}\right)$ máximo de $960 \mathrm{cSt}$ & $\left(60{ }^{\circ} \mathrm{C}\right) \mathrm{máximo} \mathrm{de} 620 \mathrm{cSt}$ \\
Densidade $15 / 4{ }^{\circ} \mathrm{C}$ & 0,98 a 0,99 & 0,98 a 1,02 & 0,98 a 1,02 \\
Teor de Cinzas & $\sim 0,05 \%$ & não especificado pela ANP* & não especificado pela ANP* \\
Teor de Enxofre & $\sim 1 \%$ & $\sim 0,8$ a $1 \%$ & $\sim 0,8$ a $1 \%$ \\
\hline
\end{tabular}

* ANP - Agência Nacional de Petróleo 
"Model-free kinetics", é baseado na teoria de Vyazovkin" ${ }^{23-25}$. Ambos os métodos possibilitam a determinação de parâmetros cinéticos por meio de termogravimetria e neste trabalho empregam-se os dois métodos com a finalidade de se comparar os resultados. A seguir será apresentada uma breve descrição destes modelos.

A norma ASTM E 1641 tem como base o método proposto por Flynn e Wall ${ }^{26}$, que utiliza a Equação 2

$\mathrm{E}_{\mathrm{e}}=-(\mathrm{R} / \mathrm{b}) * \Delta \log (\beta) / \Delta(1 / \mathrm{T})$

sendo $\beta$ a razão de aquecimento, $E_{e}$ a energia de ativação estimada, b uma variável de interação, $R$ a constante universal dos gases e $T$ a temperatura. Calcula-se a energia de ativação estimada, utilizando a Equação 2, empregando-se o valor da inclinação da reta, $\Delta(\log \beta) / \Delta(1 / \mathrm{T})$ e fixando o valor de $0,457 / \mathrm{K}$ para b na primeira interação. Obtém-se assim um primeiro valor para $\mathrm{E}_{\mathrm{e}}$. A partir deste, calcula-se $\mathrm{E}_{\mathrm{e}} / \mathrm{RT}$ e aplica-se um novo valor de b para a Equação 2. Para as interações subseqüentes utilizam-se valores de b tabelados por Doyle ${ }^{27,28}$. O cálculo de $\mathrm{E}$ é feito para cada grau de conversão e é repetido diversas vezes, alterando-se o valor de $\mathrm{b}$ até que $\mathrm{E}_{\mathrm{a}}$ atinja uma diferença menor que $1 \%$ entre os resultados finais. Portanto, esse valor final pode ser chamado energia de ativação $\mathrm{E}_{\mathrm{a}}$

O "Model-free kinetics" aplica técnicas isoconversionais para o cálculo da energia de ativação efetiva $(E)$ como uma função da conversão $(\alpha)$ da reação química, $E=f(\alpha)$. Assim, em sua aproximação seguem-se todas as conversões obtidas a partir de múltiplos experimentos. Esta teoria tem como base a Equação 3:

$\frac{\mathrm{d} \alpha}{\mathrm{dt}}=\mathrm{k}(\mathrm{T}) \mathrm{f}(\alpha)$

sendo $t$ o tempo, $T$ a temperatura e $\alpha$ a extensão de conversão, $f(\alpha)$ representa o modelo de reação e $k(T)$ o coeficiente taxa de reação de Arrhenius, então:

$\frac{\mathrm{d} \alpha}{\mathrm{dt}}=\operatorname{Aexp}\left(\frac{-\mathrm{E}}{\mathrm{RT}}\right) \mathrm{f}(\alpha)$

sendo $\mathrm{R}$ a constante universal dos gases.

Tomando-se como base a Equação 3 e dividindo pela razão de aquecimento $\beta=\mathrm{dT} / \mathrm{dt}$ obtém-se,

$$
\frac{\mathrm{d} \alpha}{\mathrm{dt}}=\mathrm{kf}(\alpha) \Rightarrow \frac{\mathrm{d} \alpha}{\mathrm{dT}}=\frac{\mathrm{k}}{\beta} \mathrm{f}(\alpha)
$$

sendo d $\alpha / \mathrm{dt}$ a taxa de reação $\left(\mathrm{s}^{-1}\right), \mathrm{k}$ a constante de velocidade $\left(\mathrm{s}^{-1}\right)$.

Substituindo a Equação 5 na Equação 4, têm-se:

$\frac{1}{f(\alpha)} \mathrm{d} \alpha=\frac{\mathrm{A}}{\beta} \mathrm{e}^{-\mathrm{E} / \mathrm{RT}} \mathrm{dT}$

integrando até a conversão, $\alpha$ (na temperatura T),

$\int_{0}^{\alpha} \frac{1}{f(\alpha)} d \alpha=g(\alpha)=\frac{A}{\beta} e^{-E / R T} d T$

desde que E/2RT $>>1$, a integral da temperatura poderá ser aproximada por

$\int_{T_{0}}^{\mathrm{T}} \mathrm{e}^{-\mathrm{E} / \mathrm{RT}} \mathrm{dt} \approx \frac{\mathrm{R}}{\mathrm{E}} \mathrm{T}^{2} \mathrm{e}^{-\mathrm{E} / \mathrm{RT}}$

A Equação 8 é definida como equação dinâmica, que é usada para a determinação da energia de ativação para todos os valores de conversão.

Substituindo-se a integral da temperatura e rearranjando na forma logarítmica, obtém-se:

$\ln \frac{\beta}{\mathrm{T}_{\alpha}^{2}}=\ln \left[\frac{\mathrm{RA}}{\mathrm{E}_{\alpha} \mathrm{g}(\alpha)}\right]-\frac{\mathrm{E}_{\alpha}}{\mathrm{R}_{\alpha}} \frac{1}{\mathrm{~T}_{\alpha}}$

sendo,

$\int_{0}^{\alpha} \frac{1}{f(\alpha)}=g(\alpha)$

Uma das principais vantagens deste método é a possibilidade de isolar a função $\mathrm{g}(\alpha)$ do coeficiente linear, pois a determinação desta função em processos complexos é difícil de ser determinada ${ }^{24}$.

\section{RESULTADOS E DISCUSSÃO}

As Figuras 1, 2 e 3 ilustram as curvas TG e DTG para as amostras A, B e C, na razão de aquecimento de $20{ }^{\circ} \mathrm{C} \mathrm{min}^{-1}$. Observa-se que cada amostra apresenta um perfil de curvas TG/DTG diferente, contudo, para todas elas foram identificadas três regiões distintas. Segundo a literatura ${ }^{7,8,13}$, a primeira região é denominada oxidação à baixa temperatura, LTO, e ocorre até $390{ }^{\circ} \mathrm{C}$. A segunda transição ocorre entre 390 e $490{ }^{\circ} \mathrm{C}$ e é denominada depósito de combustível, FD. A última região encontra-se entre 490 e $600{ }^{\circ} \mathrm{C}$ e é chamada de oxidação à alta temperatura, HTO.

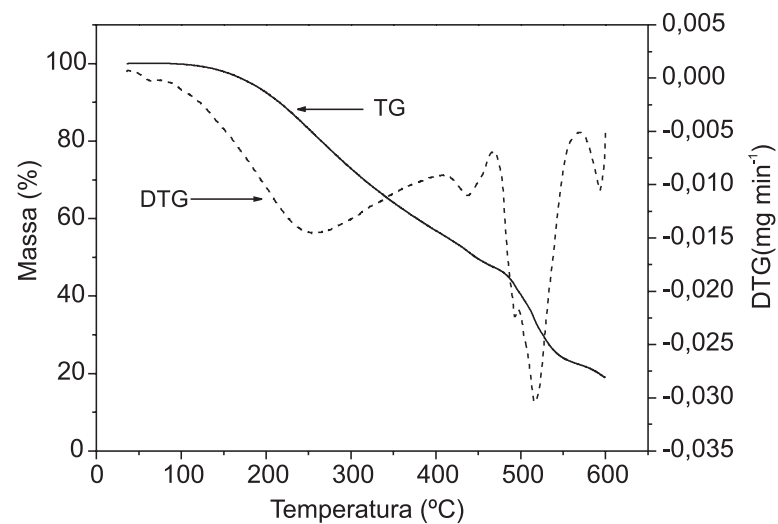

Figura 1. Curvas TG/DTG, amostra A, massa: $20 \mathrm{mg}$, razão de aquecimento: $20{ }^{\circ} \mathrm{C} \mathrm{min}^{-1}$, gás de arraste: ar sintético, vazão: $100 \mathrm{~mL} \mathrm{~min}^{-1}$

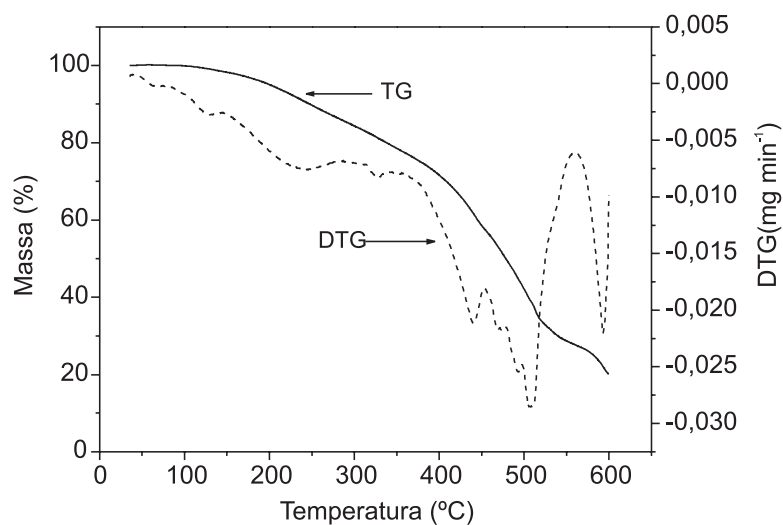

Figura 2. Curvas TG/DTG, amostra B, massa: $20 \mathrm{mg}$, razão de aquecimento: $20{ }^{\circ} \mathrm{C} \mathrm{min}^{-1}$, gás de arraste: ar sintético, vazão: $100 \mathrm{~mL} \mathrm{~min}^{-1}$ 


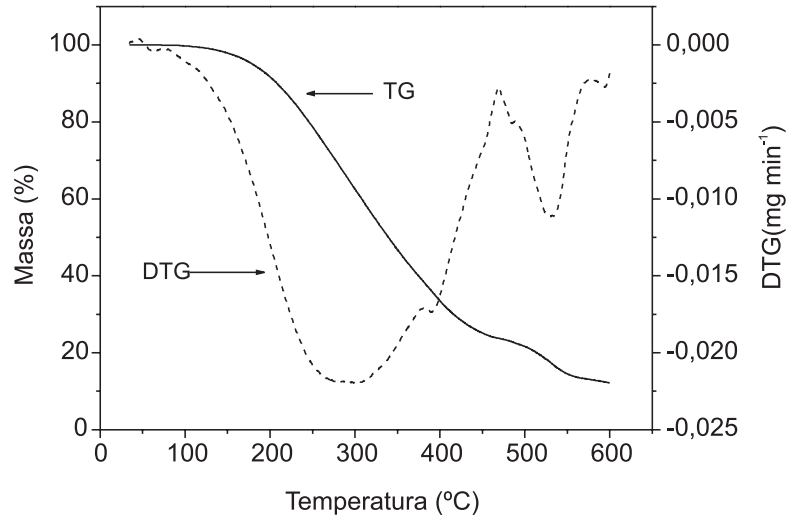

Figura 3. Curvas TG/DTG, amostra C, massa: $20 \mathrm{mg}$, razão de aquecimento: $20{ }^{\circ} \mathrm{C} \mathrm{min}^{-1}$, gás de arraste: ar sintético, vazão: $100 \mathrm{~mL} \mathrm{~min}^{-1}$

O "Model-free kinetics" requer pelo menos três curvas dinâmicas com diferentes razões de aquecimento e o método ASTM requer pelo menos quatro razões de aquecimento. Neste trabalho foram utilizadas as cinco razões de aquecimento para ambos os modelos. As Figuras 4, 5 e 6 mostram as curvas TG das amostras A, B e C para as cinco razões de aquecimento.

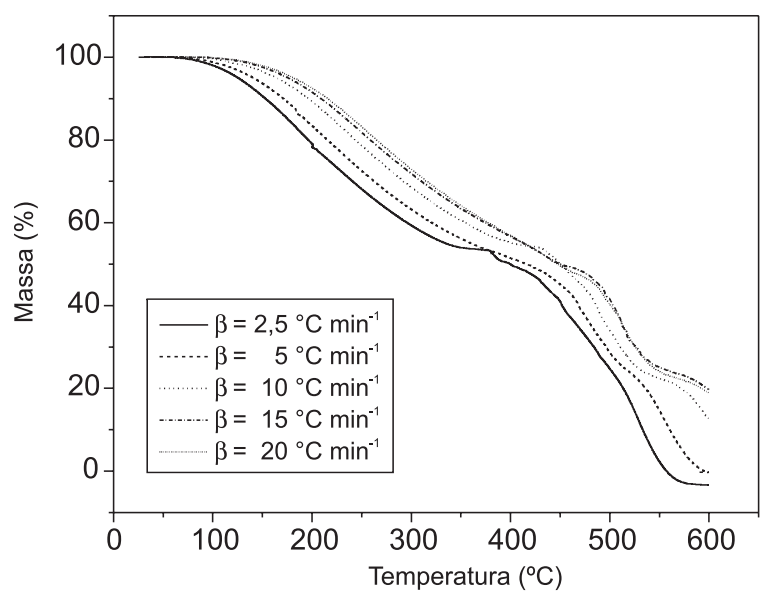

Figura 4. Curva TG, amostra A, cinco razões de aquecimento: 2,5; 5,0; 10,0; 15,0 e $20,0^{\circ} \mathrm{C} \mathrm{min}^{-1}$

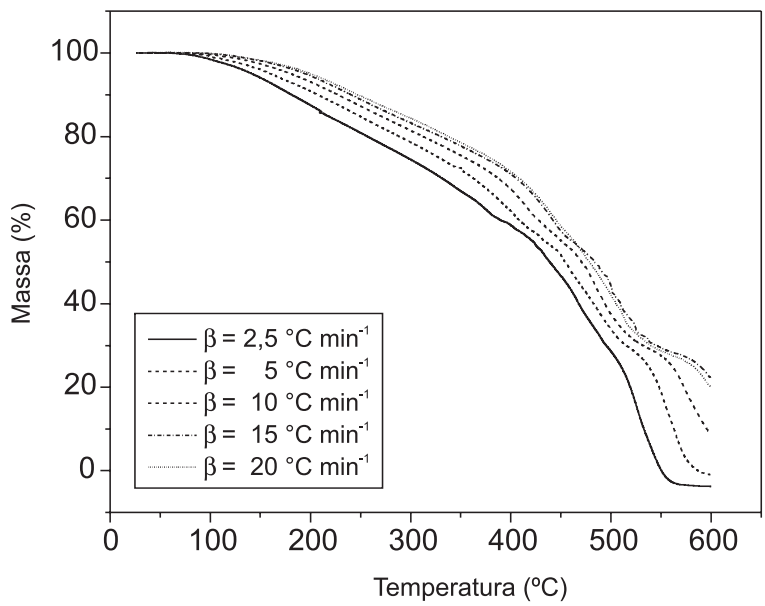

Figura 5. Curva TG, amostra B, cinco razões de aquecimento: 2,5; 5,0; 10,0; 15,0 e $20,0{ }^{\circ} \mathrm{C} \mathrm{min}^{-1}$

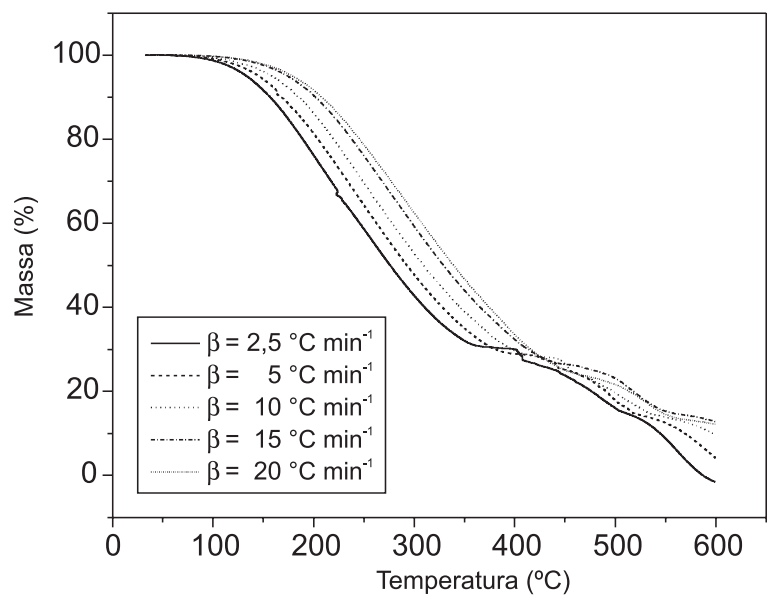

Figura 6. Curva TG, amostra C, cinco razões de aquecimento: 2,5; 5,0; 10,0; 15,0 e $20,0^{\circ} \mathrm{C} \mathrm{min}^{-1}$

Para se determinar os parâmetros cinéticos, tanto pelo método ASTM quanto pelo "Model-free kinetics", avaliaram-se primeiramente os graus de conversão de um determinado evento com base na Equação 11

$\alpha=\frac{\mathrm{m}-\mathrm{m}_{0}}{\mathrm{~m}_{\infty}-\mathrm{m}_{0}}$

sendo $\alpha$ a conversão, $m$ a massa da amostra que varia com o tempo (t), $m_{0}$ a massa inicial da amostra e $m_{\infty}$ a massa remanescente.

Optou-se por se estudar a região de oxidação à baixa temperatura (LTO), cuja perda de massa corresponde à liberação de voláteis (hidrocarbonetos de baixa massa molar) e águas de hidratação ${ }^{11}$. As conversões foram calculadas a partir das curvas TG desta região.

As Figuras 7, 8 e 9 mostram as curvas de conversão em função da temperatura.

Para o cálculo da energia de ativação pelo método ASTM foram escolhidos níveis de decomposição de 10,0; 20,0; 30,0; 40,0; 50,0; 60,0 e $80,0 \%$. Representou-se graficamente o logaritmo da razão de aquecimento $(\log \beta)$ versus o inverso da temperatura correspondente à conversão $\alpha\left(1 / \mathrm{T}_{\alpha}\right)$ a partir das cinco curvas mostradas nas Figuras 7 a 9, para as amostras A, B e C. Assim, foi obtida uma série de retas (representadas nas Figuras 10, 11 e 12), sendo que a inclinação de cada reta corresponde a $-\mathrm{E}_{\mathrm{a}} / \mathrm{R}\left(\mathrm{R}=8,314 \mathrm{~J} \mathrm{~mol}^{-1} \mathrm{~K}^{-1}\right)$.

Para o método "Model-free kinetics" para cada conversão $\alpha$, $\ln \beta / \mathrm{T}_{\alpha}{ }^{2}$ foi representado graficamente como uma função de $1 / \mathrm{T}_{\alpha}$, pro-

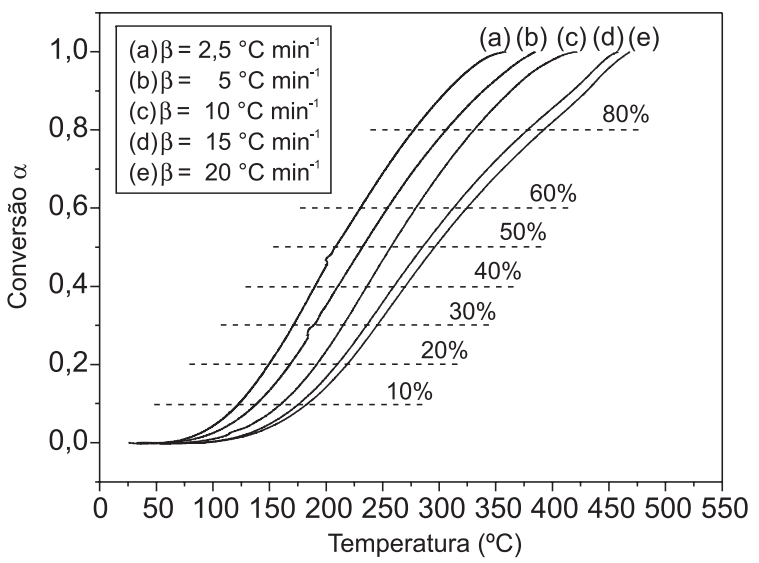

Figura 7. Curvas de conversão em função da temperatura para amostra $A$ $\left(0<a<100 b=2,5 ; 5,0 ; 10,0 ; 15,0\right.$ e $20,0{ }^{\circ} \mathrm{C} \mathrm{min}^{-1}$ 


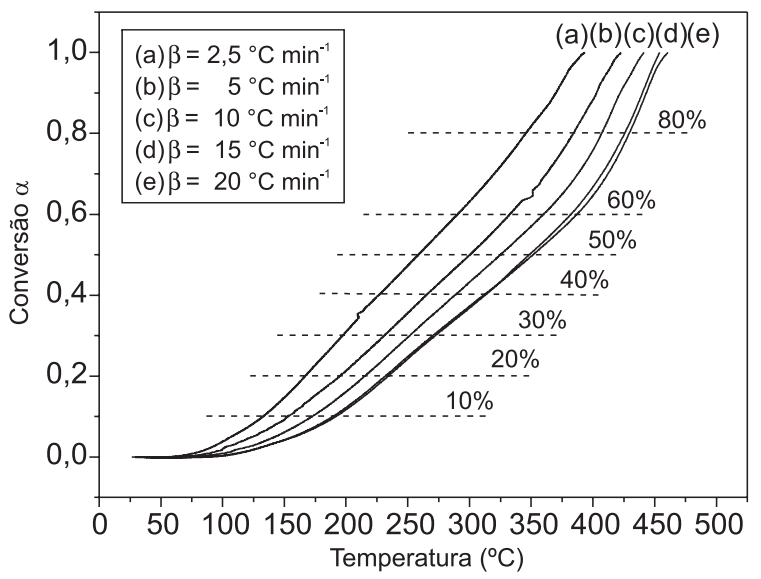

Figura 8. Curvas de conversão em função da temperatura para amostra $B$ $(0<a<100), b=2,5 ; 5,0 ; 10,0 ; 15,0$ e $20,0{ }^{\circ} \mathrm{C} \mathrm{min}^{-1}$

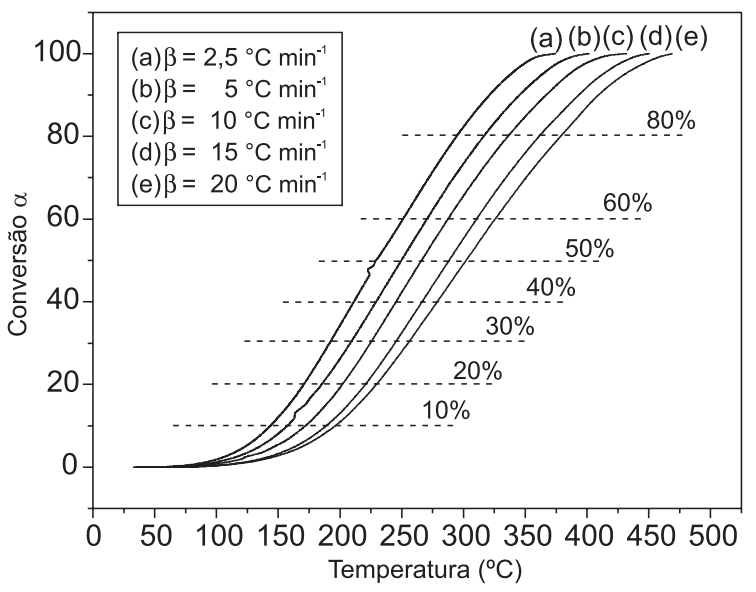

Figura 9. Curvas de conversão em função da temperatura para amostra $C$ $(0<a<100), b=2,5 ; 5,0 ; 10,0 ; 15,0$ e $20,0{ }^{\circ} \mathrm{C} \mathrm{min}^{-1}$

porcionando também uma série de retas com a inclinação $-\mathrm{E}_{\alpha} / \mathrm{R}$, deste modo, a energia de ativação foi obtida em função da conversão.

Os valores de energia de ativação obtidos em ambos os métodos estão mostrados na Tabela 2.

Por meio da regressão linear é possível estabelecer a $\mathrm{E}_{\alpha}$ para toda a extensão de conversão. As Figuras 13, 14 e 15 ilustram os conjuntos destas $\mathrm{E}_{\alpha}$ obtidas para as amostras $\mathrm{A}, \mathrm{B}$ e $\mathrm{C}$, tanto pelo método ASTM quanto pelo "Model-free kinetics". Nota-se que a $\mathrm{E}_{\alpha}$ varia com a conversão e os perfis das curvas são característicos para cada amostra.

Observa-se que os valores de energia de ativação para ambos os métodos são muito próximos, e isto sugere que tanto o método ASTM quanto o "Model-free kinetics" são adequados para a determinação da energia de ativação.

Como mencionado anteriormente, a região escolhida para a determinação da energia de ativação corresponde ao processo de oxidação à baixa temperatura (LTO), sendo este caracterizado por um evento exotérmico. A técnica DTA caracteriza este evento e a área sob a curva DTA é proporcional à entalpia do processo, entretanto, este valor pode ser usado para avaliar semiquantitativamente o calor de reação ${ }^{29}$.

As Figuras 16, 17 e 18 mostram as curvas DTA na região LTO das amostras A, B e C. Notam-se picos exotérmicos pronunciados em torno de 384,366 e $340{ }^{\circ} \mathrm{C}$, respectivamente.

As áreas correspondentes às curvas DTA da região LTO das

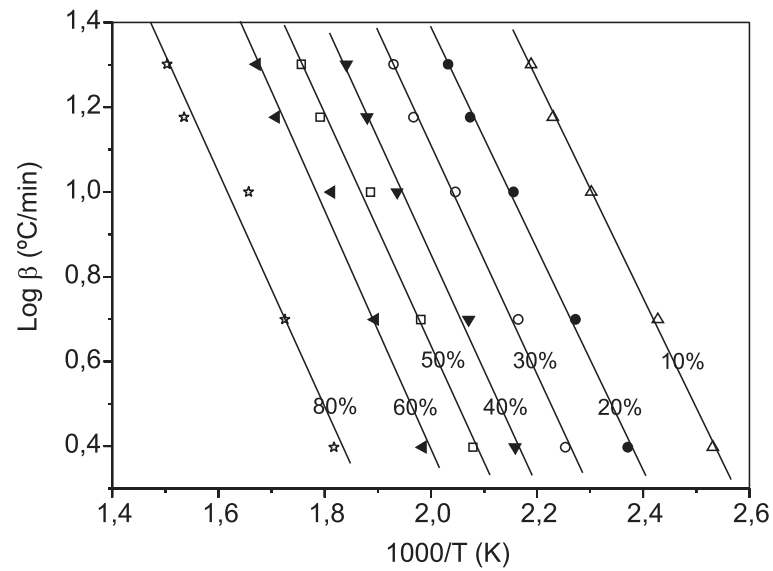

Figura 10. Combustão do óleo combustível: logaritmo da razão de aquecimento versus $1000 / K$ correspondente à conversão a $\left(1 / T_{a}\right)$ em diferentes níveis de conversão $(10,0 ; 20,0 ; 30,0 ; 40,0 ; 50,0 ; 60,0$ e 80,0\%) para a amostra A

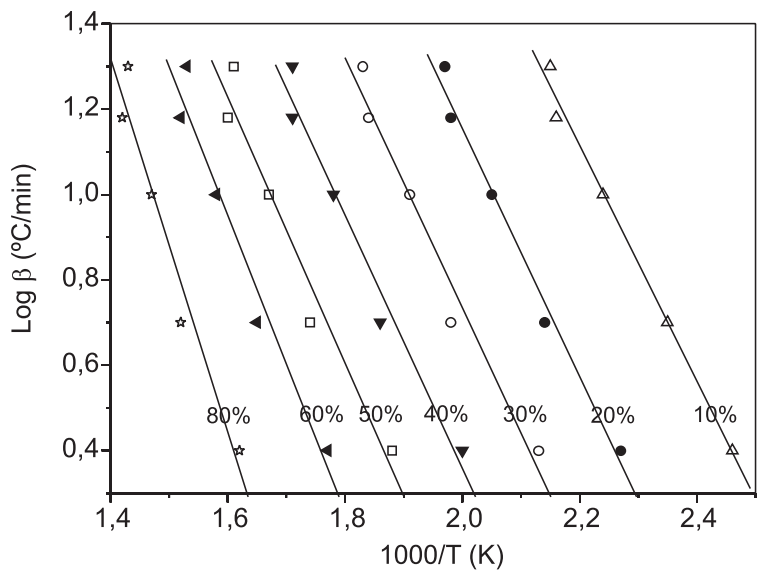

Figura 11. Combustão do óleo combustível: logaritmo razão de aquecimento versus 1000/K correspondente à conversão a $\left(1 / T_{a}\right)$ em diferentes níveis de conversão $(10,0 ; 20,0 ; 30,0 ; 40,0 ; 50,0 ; 60,0$ e 80,0\%) para a amostra $B$

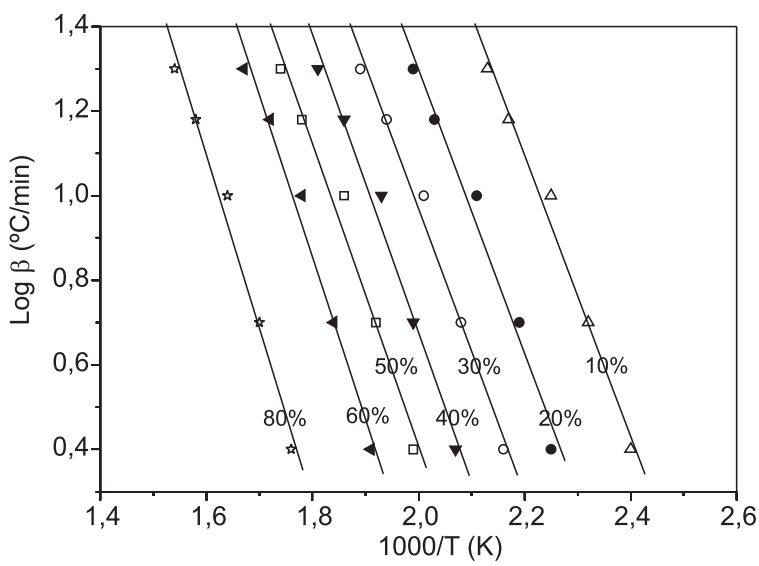

Figura 12. Combustão do óleo combustível: logaritmo razão de aquecimento versus $1000 / K$ correspondente à conversão a $\left(1 / T_{a}\right)$ em diferentes níveis de conversão $(10,0 ; 20,0 ; 30,0 ; 40,0 ; 50,0 ; 60,0$ e 80,0\%) para a amostra $C$

amostras de óleo combustível foram amostra $\mathrm{A}=817 \mu \mathrm{V}{ }^{\circ} \mathrm{C}$; amostra $\mathrm{B}=836 \mu \mathrm{V}{ }^{\circ} \mathrm{C}$ e amostra $\mathrm{C}=916 \mu \mathrm{V}{ }^{\circ} \mathrm{C}$, obtidas pelo método integral.

Quanto à energia de ativação desta mesma região estudada es- 
Tabela 2. Energias de ativação para as conversões de 10,0; 20,0; 30,0; 40,0; 50,0; 60,0 e 80,0\% para as amostras A, B e C obtidas pelo métodos ASTM E 1641 e "Model-free kinetics"

\begin{tabular}{|c|c|c|c|c|c|c|}
\hline \multirow[b]{3}{*}{ Conversão (\%) } & \multicolumn{2}{|c|}{ AMOSTRA A } & \multicolumn{2}{|c|}{ AMOSTRA B } & \multicolumn{2}{|c|}{ AMOSTRA C } \\
\hline & ASTM & Model-free & ASTM & Model-free & ASTM & Model-free \\
\hline & $\mathrm{E}_{\mathrm{a}}(\mathrm{kJ} / \mathrm{mol})$ & $\mathrm{E}_{\mathrm{a}}(\mathrm{kJ} / \mathrm{mol})$ & $\mathrm{E}_{\mathrm{a}}(\mathrm{kJ} / \mathrm{mol})$ & $\mathrm{E}_{\mathrm{a}}(\mathrm{kJ} / \mathrm{mol})$ & $\mathrm{E}_{\mathrm{a}}(\mathrm{kJ} / \mathrm{mol})$ & $\mathrm{E}_{\mathrm{a}}(\mathrm{kJ} / \mathrm{mol})$ \\
\hline 10 & 43,44 & 43,11 & 48,00 & 45,49 & 56,03 & 55,57 \\
\hline 20 & 43,52 & 43,28 & 49,42 & 47,63 & 57,58 & 56,35 \\
\hline 30 & 45,43 & 43,74 & 50,40 & 48,60 & 57,33 & 58,18 \\
\hline 40 & 45,94 & 44,63 & 49,82 & 47,49 & 58,64 & 59,85 \\
\hline 50 & 44,95 & 44,13 & 54,00 & 50,40 & 59,62 & 60,01 \\
\hline 60 & 46,45 & 44,61 & 59,41 & 54,20 & 63,67 & 63,60 \\
\hline 80 & 45,50 & 43,02 & 82,66 & 75,69 & 65,97 & 66,23 \\
\hline
\end{tabular}

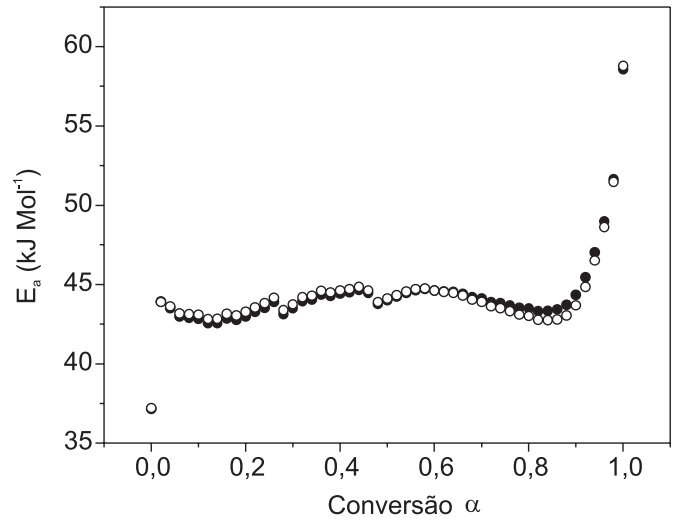

Figura 13. Comparação E versus a, amostra $A, \beta=2,5 ; 5,0 ; 10,0 ; 15,0 e$ 20,0 ${ }^{\circ} \mathrm{C} \mathrm{min}^{-1}$, (•) ASTM; (O)Model Free

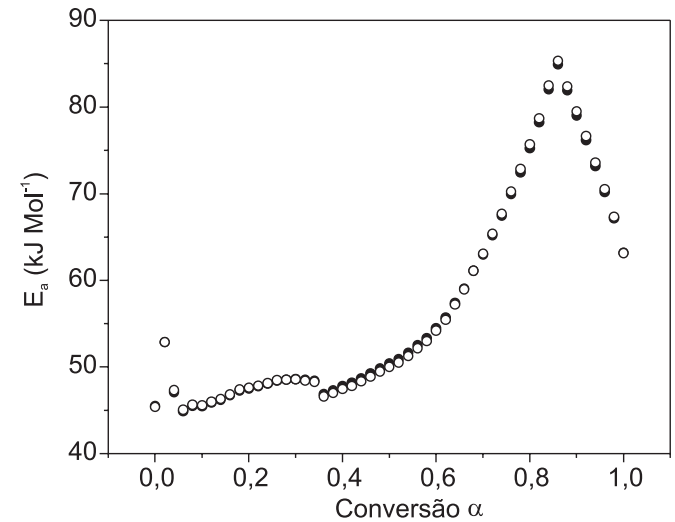

Figura 14. Comparação E versus a, amostra $B, \beta=2,5 ; 5,0 ; 10,0 ; 15,0$ e 20,0 ${ }^{\circ} \mathrm{C} \mathrm{min}^{-1}$, (•) ASTM; (O)Model Free

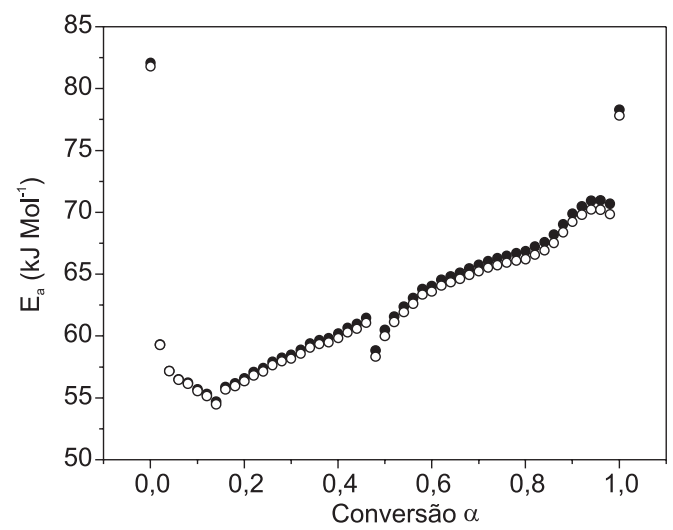

Figura 15. Comparação E versus a, amostra $C, \beta=2,5 ; 5,0 ; 10,0 ; 15,0$ e 20,0 ${ }^{\circ} \mathrm{C} \mathrm{min}^{-1}$, (•) ASTM; (O)Model Free

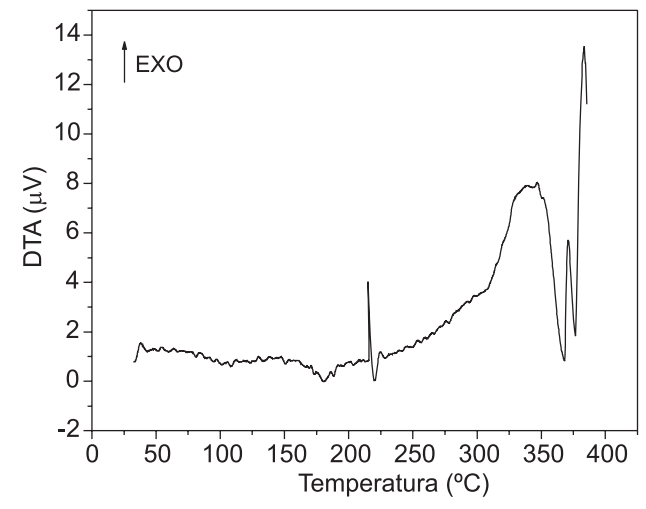

Figura 16. Curva DTA, amostra A, massa: $20 \mathrm{mg}$, razão de aquecimento: 5, ${ }^{\circ} \mathrm{C} \mathrm{min}^{-1}$, gás de arraste: ar sintético, vazão: $100 \mathrm{~mL} \mathrm{~min}^{-1}$

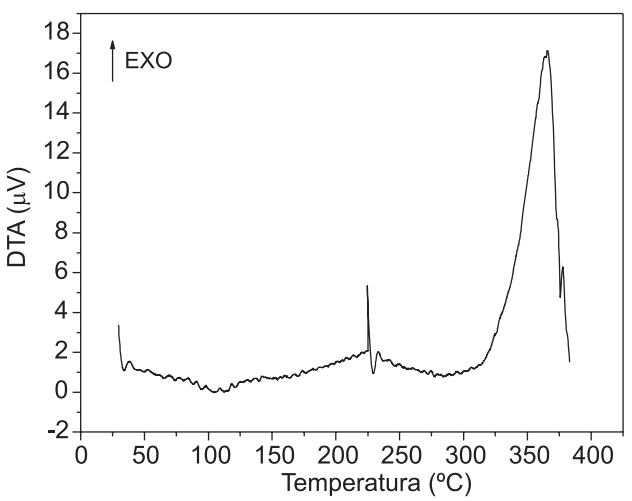

Figura 17. Curva DTA, amostra B, massa: 20 mg, razão de aquecimento: $5,0^{\circ} \mathrm{C} \mathrm{min}^{-1}$, gás de arraste: ar sintético, vazão: $100 \mathrm{~mL} \mathrm{~min}^{-1}$

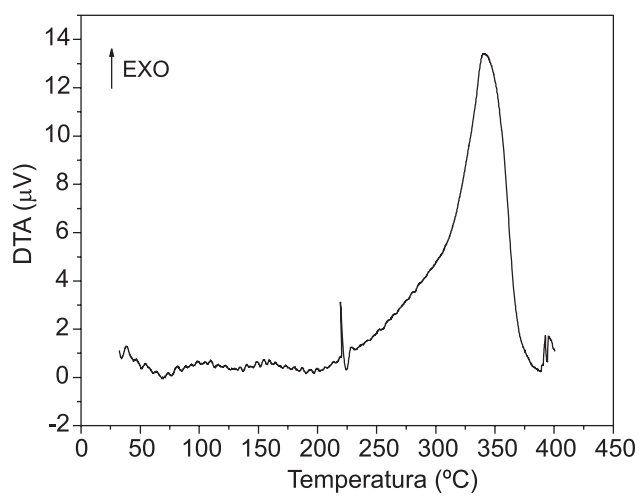

Figura 18. Curva DTA, amostra C, massa: $20 \mathrm{mg}$, razão de aquecimento: $5,0{ }^{\circ} \mathrm{C} \mathrm{min}^{-1}$, gás de arraste: ar sintético, vazão: $100 \mathrm{~mL} \mathrm{~min}^{-1}$ 
tabeleceu-se o valor médio a partir de todos aqueles encontrados entre 0 e $100 \%$. Pelo método ASTM foram determinados os seguintes valores: amostra $\mathrm{A}=44,3 \mathrm{~kJ} \mathrm{~mol}^{-1}$, amostra $\mathrm{B}=56,6 \mathrm{~kJ}$ $\mathrm{mol}^{-1} \mathrm{e}$ amostra $\mathrm{C}=61,9 \mathrm{~kJ} \mathrm{~mol}^{-1}$. Pelo "Model-free kinetics" obtiveram-se os valores: amostra $\mathrm{A}=44,1 \mathrm{~kJ} \mathrm{~mol}^{-1}$, amostra $\mathrm{B}=57,0$ $\mathrm{kJ} \mathrm{mol}^{-1}$ e amostra $\mathrm{C}=63,0 \mathrm{~kJ} \mathrm{~mol}^{-1}$.

\section{CONCLUSÃO}

Os valores de $\mathrm{E}_{\mathrm{a}}$ para os métodos aplicados são muito próximos, sugerindo que tanto o método ASTM quanto o "Model-free kinetics" são adequados para se determinar este parâmetro cinético relacionado à combustão de óleos. Observa-se que a $\mathrm{E}_{\mathrm{a}}$ é diferente de uma amostra para a outra e que está correlacionada diretamente com a exotermicidade do evento (área sob a curva DTA), ou seja, quanto menor $\mathrm{E}_{\mathrm{a}}$ menor o calor envolvido na reação.

Estes parâmetros relacionados à degradação térmica oxidativa, além de diferenciarem um óleo de outro, sugerem comportamentos diferentes, principalmente em relação à qualidade da combustão.

De acordo com a Equação 1 menor será o atraso de ignição, quanto menor for a $\mathrm{E}_{\mathrm{a}} \mathrm{e}$, conseqüentemente, melhor será o combustível. Partindo deste princípio e considerando-se apenas a primeira região de oxidação (LTO), dentre os óleos estudados, a amostra A (MF 380) apresenta-se potencialmente como melhor opção para o processo de combustão.

\section{AGRADECIMENTOS}

Ao suporte financeiro e às bolsas concedidas pela CAPES e FAPESP, à Petrobras - CENPES pelo fornecimento das amostras.

\section{REFERÊNCIAS}

1. Perrone, R. C.; Introdução à refinação de petróleo, CENAP - Petrobras: Rio de Janeiro, 1965.

2. Heywood, J.; Internal Combustion Engine Fundamentals, McGraw-Hill: New York, 1988

3. Obert, E. F.; Motores de Combustão Interna, Globo: Porto Alegre, 1971.

4. Lichty, L. C.; Combustion Engine Process, Mc Graw-Hill: New York, 1967.

5. Drici, O.; Vossoughi, S.; J. Pet. Tech. 1985, April, 731.

6. Verkoczy, B.; Jha, K. N.; J. Can. Pet. Tech. 1986, May-June, 47.

7. Kök, M. V.; Thermochim. Acta 1993, 214, 315.

8. Kök, M. V.; Hughes, R.; Price, D.; Thermochim. Acta 1996, 287, 91.

9. Kök, M. V.; Okandan, E.; J. Thermal Anal. 1997, 48, 343.

10. Kök, M. V.; Karacan, O.; J. Thermal Anal. 1998, 52, 781.

11. Ali, M. A.; Siddiqui, M. A. B.; Zaidi, S. M. J.; J. Thermal Anal. 1998, 51, 307.

12. Kök, M. V.; Thermochim. Acta 2001, 369, 149

13. Kök, M. V.; Keskin, C.; Thermochim. Acta 2001, 369, 143.

14. Gonçalves, M. L. A.; Teixeira, M. A. G.; Pereira, R. C. L.; Mercury, R. L. P.; Matos, J. R.; J. Therm. Anal. Calorim. 2001, 64, 697.

15. Kök, M. V.; J. Therm. Anal. Calorim. 2003, 73, 241.

16. Kök, M. V.; Sztatisz, J.; Pokol, G.; Energy Fuels 1997, 11, 1137.

17. Mackenzie, R. C.; Thermochim. Acta 1979, $28,1$.

18. Burnham, A. K.; Braun, R. L.; Energy Fuel 1999, 13, 1.

19. Tadema H. J., Proc. World Pet. Congr., $5^{\text {th }}$, Section II, Paper, 1956, 22, 279 apud Kök, M. V.; Thermochim. Acta 1993, 214, 315.

20. Millington, A.; Price, D.; Hughes, R.; J. Therm. Anal. 1993, 40, 225.

21. Kök, M. V.; J. Therm. Anal. Calorim. 2002, 68, 1061.

22. ASTM E $1641 / 99$.

23. Vyazovkin, S.; Wight, C. A.; Thermochim. Acta 1999, 340-341, 53.

24. Vyazovkin, S.; Sbirrazzuoli, N.; Anal. Chim. Acta 1997, 355, 175.

25. Vyazovkin, S.; Dollimore, D.; J. Chem. Inf. Comput. Sci. 1996, 36, 42.

26. Flynn, J. H.; Wall, L. A.; Polym. Lett. 1966, 4, 323.

27. Doyle, C. D.; J. Appl. Polym. Sci. 1961, 5, 285.

28. Doyle, C. D.; J. Appl. Polym. Sci. 1962, 6, 639.

29. Wendlandt, W.; Thermal Analysis, Jonh Wiley: New York, 1986. 